\title{
ANÁLISIS AUTOMATIZADO DE ENCUADRES MEDIÁTICOS. COBERTURA EN PRENSA DEL DEBATE 7D 2015: EL DEBATE DECISIVO
}

\author{
Automated analysis of media frames. Press coverage \\ of the 7D 2015 debate: The decisive debate
}

Vicente Fenoll y Paula Rodríguez-Ballesteros

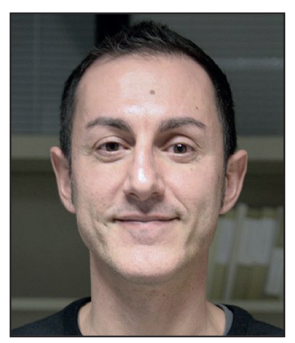

Vicente Fenoll es profesor de Comunicación audiovisual en la Universitat de València. Tiene una larga experiencia profesional en televisión y en el sector audiovisual. Su investigación se centra en los estudios de encuadre, el análisis de la comunicación digital y los efectos de las crisis y las dictaduras en la producción cultural. Ha realizado estancias de investigación en universidades americanas y europeas.

http://mediaflows.es/investigador/vicente-fenoll

http://orcid.org/0000-0002-5851-4237

vicente.fenoll@uv.es

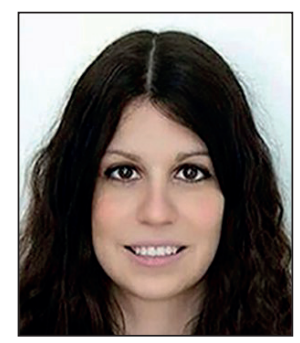

Paula Rodríguez-Ballesteros es graduada en Periodismo y Comunicación audiovisual por la Universitat de València (UV). Cursa el máster Interculturalidad, comunicación y estudios europeos de la UV. Ha realizado prácticas en medios digitales como La vanguardia digital y Summon Press (beca Santander), y en radio (Mediauni). Ha disfrutado de una beca de iniciación a la investigación en el marco del proyecto de I+D "Los flujos de comunicación en los procesos de movilización política: medios, blogs y líderes de opinión". http://mediaflows.es/investigador/paula-rodriguez http://orcid.org/0000-0001-6585-6430

pauroba@alumni.uv.es

Universitat de València, Facultad de Filología, Traducción y Comunicación Dpto. de Teoría de los Lenguajes y Ciencias de la Comunicación Av. Blasco Ibáñez, 32, planta 5ạ. 46010 Valencia, España

\section{Resumen}

La digitalización y el almacenamiento de las noticias en bases de datos favorece la investigación de grandes lotes de información textual mediante el análisis de contenido asistido por computadora. Varios autores señalan las ventajas de la utilización de este tipo de programas en los estudios de framing. El objetivo de este trabajo es analizar la presencia en los medios de comunicación de los encuadres utilizados por los participantes en el debate electoral televisado el 7 de diciembre de 2015 con motivo de las elecciones generales españolas de 2015. En el estudio implementamos un análisis automatizado de contenido con el programa WordStat para extraer los encuadres de los textos analizados, a través de una metodología que combina técnicas cuantitativas y cualitativas. Los resultados del estudio muestran que, pese a producirse un incremento notable en el número de noticias protagonizadas por los candidatos tras el debate, la cobertura en prensa no informa sobre todos los temas abordados en el mismo y presenta a los políticos como si participaran en una carrera de caballos.

\section{Palabras clave}

Framing; Análisis computerizado de contenidos; Debate electoral; Elecciones generales España 2015; WordStat.

\section{Abstract}

The digitization and storage of news in databases facilitates the research of large amounts of text when using computer assisted content analysis techniques. Several authors point out the advantages of using computer programs in framing studies. The aim of this paper is to analyze the presence in the media of frames used by participants in the electoral debate televised on December 7, 2015 on the occasion of the Spanish general elections of 2015. In our study we implemented an automated analysis content with WordStat software to extract the frames of the texts analyzed, through a method that combines quantitative and qualitative techniques. The results of the study show that, in spite of a noticeable increase in the number 
of news articles about the candidates after the debate, the press coverage does not report on all the topics covered in the debate and presents politicians as if they participated in a horse race.

\section{Keywords}

Framing; Computer-assisted content analysis; Presidential debate; Spanish general election 2015; WordStat.

Fenoll, Vicente; Rodríguez-Ballesteros, Paula (2017). "Análisis automatizado de encuadres mediáticos. Cobertura en prensa del debate 7D 2015: el debate decisivo". El profesional de la información, v. 26, n. 4, pp. 630-640.

https://doi.org/10.3145/epi.2017.jul.07

\section{Introducción}

El 20 de diciembre de 2015 tuvieron lugar las duodécimas elecciones generales en España, que han cobrado gran relevancia por suponer la ruptura del sistema bipartito y por la irrupción por primera vez a nivel nacional de los partidos Ciudadanos y Podemos. Durante la campaña electoral tuvo especial relevancia la cobertura por parte de los medios de los candidatos de los partidos emergentes porque todas las encuestas les situaban dentro del Parlamento, disputándose incluso la segunda posición.

En este contexto, despertó gran interés el debate televisado del 7 de diciembre, 7D: El debate decisivo, en el que participaron los candidatos a la presidencia del gobierno de tres de los cuatro principales partidos políticos: Pablo Iglesias por Podemos, Pedro Sánchez por el Partido Socialista Obrero Español (PSOE) y Albert Rivera por Ciudadanos, junto con Soraya Sáenz de Santamaría por el Partido Popular (PP). Cabe destacar que Soraya Sáenz de Santamaría acudió en representación de Mariano Rajoy que, pese a ser el candidato del $P P$, rechazó la propuesta de acudir al debate.

Se trató de un debate de especial relevancia porque se confrontaban las posiciones de los representantes de los cuatro partidos con mayor peso en las encuestas en un momento en que un $41,6 \%$ de los electores todavía no había decidido su elección de voto (CIS, 2015). De este modo, al tratarse de uno de los últimos actos de campaña en el que los candidatos muestran sus propuestas y posiciones, la cobertura del debate en los medios cobra especial importancia. El presente trabajo tiene como objetivo extraer los encuadres utilizados por los cuatro participantes en el debate electoral del 7D para comprobar su impacto en la cobertura mediática. Para ello, vamos a aplicar la metodología del frame mapping (Miller, 1997), mediante un análisis de texto automatizado con el programa WordStat.

A continuación, repasaremos el papel del encuadre en los procesos electorales tanto desde la perspectiva de los candidatos como de los medios de comunicación. Seguidamente, veremos las técnicas de análisis de texto automatizado utilizadas en los estudios de framing para la extracción de encuadres. A partir de esta conceptualización teórica y empírica, definiremos el planteamiento metodológico utilizado para la consecución de nuestros objetivos. Tras la presentación y análisis de los resultados de la investigación, procederemos a la exposición de las conclusiones.

\section{Framing en procesos electorales}

Los estudios de framing tienen sus raíces en campos como la sociología y la psicología, así como en estudios políticos y mediáticos (De-Vreese, 2012; López-Rabadán, 2010; Matthes, 2014; Miller; Riechert, 2001; Pan; Kosicki, 1993; Touri; Koteyko, 2015; Vicente-Mariño; López-Rabadán, 2009). Según la definición de Robert Entman:

"Encuadrar es seleccionar algunos aspectos de la realidad percibida y hacerlos más relevantes en un texto comunicativo, de modo que promuevan definiciones particulares de los problemas, interpretaciones causales, evaluaciones morales y/o recomendaciones para el tratamiento del asunto descrito" (Entman, 1993, p. 52).

Es decir, seleccionar, enfatizar y estructurar paquetes de información para fijar una perspectiva desde la que interpretar un tema.

En las campañas electorales los candidatos aprovechan los debates para desplegar sus "paquetes interpretativos" (Gamson; Modigliani, 1989, p. 3) o "paquetes de argumentos temáticos consistentes" (Matthes, 2012, p. 254), con los que encuadrar los temas políticos tratados (Chihu, 2011). Los paquetes de encuadre están compuestos por 3 elementos:

- el núcleo del encuadre, que es el que lo define como un todo;

- los dispositivos de encuadre, que son las frases, metáforas o descripciones utilizadas en su elaboración; y

- los dispositivos de razonamiento, que están relacionados con las consideraciones causales que pueden ser evocadas cuando el objeto encuadrado se asocia con un marco particular que está consolidado en una cultura (Gamson; Modigliani, 1989; Van-Gorp; Vercruysse, 2012).

En la información sobre la campaña, los medios pueden reproducir en su cobertura los encuadres de los candidatos (advocacy frames) o plantear un encuadre propio (journalistic news frames) (De-Vreese, 2012). Los estudios electorales señalan que los medios informan sobre los candidatos como si participaran en una carrera de caballos (Aalberg; Strömback; De-Vreese, 2011; Iyengar; Norpoth; Hahn, 2004; Patterson, 1994), con predominio de informaciones que utilizan el denominado encuadre estratégico o del juego (Berganza, 2008; Cappella; Jamieson, 1997; De-Vreese, 2005; Lawrence, 2010; López-Rabadán, 2010; Muñiz, 2015; Strömbäck; 
Luengo, 2008). Este tipo de encuadre se caracteriza por el uso de vocabulario bélico o deportivo para describir la posición del candidato en la carrera electoral, en contraposición al encuadre temático, que se centra en la posición de los candidatos respecto a los temas políticos en discusión o el programa de gobierno que platean (Lawrence, 2010).

En las noticias que siguen el encuadre del juego,

"los periodistas tienden a hacer énfasis en las estrategias, más que en los temas de campaña" (Chihu, 2001, p. 76).

De este modo, la cobertura electoral es más interpretativa que descriptiva y disminuye la información que transmite los planteamientos que manifiestan los políticos:

"mientras la información descriptiva está dirigida por los hechos, el modo interpretativo está dirigido por el tema sobre el que la historia se construye" (Patterson, 1996, p. 102).

Algunos autores señalan que la cobertura del debate puede influir en la percepción que los ciudadanos tienen de los candidatos (Luengo, 2011). La forma en que los periodistas presentan la información política moldea la perspectiva con que la audiencia percibe a los candidatos y, por tanto, puede incrementar la desafección política (Cappella; Jamieson, 1997; Patterson, 1994, 1996, 1998) o influir en la decisión de voto (Cacciatore; Scheufele; Iyengar, 2016; Cappella; Jamieson, 1997; Chong; Druckman, 2007; D’Angelo, 2002; Entman, 2010; Matthes, 2012; Pan; Kosicki, 1993; Scheufele; lyengar, 2014; Tankard, 2001; Tolley, 2011). Debido a la relevancia que tiene para el sistema democrático, es pertinente comprobar en qué medida los medios reproducen en sus noticias los temas y encuadres que utilizan los políticos en los debates electorales.

\section{Análisis de contenido automatizado en estudios de framing}

El análisis de contenido automatizado permite afrontar la codificación de grandes cantidades de información de manera sistemática. Asimismo, la incorporación de cálculos estadísticos para el análisis de contenido aumenta la confiabilidad del proceso y reduce los sesgos subjetivos que pueda introducir el investigador durante la interpretación de los textos (Arcila-Calderón; Barbosa-Caro; Cabezuelo-Lorenzo, 2016; Fenoll, 2016; Miller, 1997; Miller; Andsager; Riechert, 1998; Neuendorf, 2002; Young; Soroka; 2012). No obstante, la utilización de estos programas plantea algunas dudas respecto a su validez (Harwood; Garry, 2003; Tolley, 2011), así como la dificultad para codificar adecuadamente aspectos como la ironía o el humor (Fenoll, 2015).

Dentro de las herramientas más habituales que encontramos en los programas de análisis de contenido automatizado destacan las listas de frecuencia de palabras clave, las colocaciones y las concordancias. Asimismo, existen aplicaciones basadas en diccionarios que extraen el porcentaje de palabras pertenecientes a una determinada categoría. Un diccionario puede contener dimensiones que clasifiquen las palabras según sus características lingüísticas, emocionales, cognitivas, etc., aunque también pueden incorporar categorías de palabras elaboradas ad hoc por el investigador. En el estudio se utiliza el paquete QDA Miner-WordStat, ya que permite la combinación de todas estas funciones y ha demostrado su eficiencia para llevar a cabo análisis de contenidos complejos en estudios de framing (Entman, 2010, p. 334).

El análisis de contenido automatizado incrementa la fiabilidad de la codificación, ya que

"Ios encuadres no son encontrados por el investigador sino computados por el programa" (Matthes; Kohring, 2008, p. 261).

Igualmente, dado que las categorizaciones de encuadres y los esquemas analíticos no son introducidos artificialmente desde el exterior del texto analizado, se asegura la validez del proceso (Groshek; Engelbert, 2013).

La metodología inductivo-cuantitativa para la extracción de encuadres mediante el análisis de contenido automatizado se basa en la afirmación de Entman (1993) de que encuadrar implica "seleccionar y enfatizar algunas características de la realidad y omitir otras" (p. 53) y, por tanto, "los encuadres se manifiestan por la presencia o ausencia de determinadas palabras clave" (p. 52). De este modo, los encuadres pueden detectarse mediante el sondeo de las palabras que aparecen constantemente en la narrativa de los medios para transmitir significados temáticamente consonantes (Entman, 1991, p. 7).

La mayoría de estudios que utilizan técnicas de análisis de contenido automatizado incorporan, en mayor o menor medida, la participación humana en el proceso de desarrollo del diccionario o en la interpretación de los resultados de la codificación (Fenoll, 2016; White; Marsh, 2006). Encontramos esta práctica en estudios sobre la cobertura del cambio climático (Grundmann; Krishnamurthy, 2010; Soroka et al., 2012), la representación mediática de Europa (Just, 2009), el tratamiento de la crisis financiera griega (Touri; Koteyko, 2015) o los comentarios de los usuarios de los medios digitales (Fenoll, 2015).

No obstante, la metodología de frame mapping (Luther; MiIler, 2005; Miller, 1997; Miller; Andsager; Riechert, 1998; Miller; Riechert, 2001), permite reducir todavía más la intervención del investigador en el proceso de detección y extracción de las dimensiones latentes de los encuadres, al introducir un análisis de contenido automatizado basado en cálculos estadísticos de coocurrencias (Miller, 1997).

Según Miller y Riechert (2001, pp. 107-108), individuos, grupos informales o grupos de interés organizados funcionan como partes interesadas que intentan transferir sus reivindicaciones a la opinión pública para influir en las decisiones políticas y orientarlas a favor de sus intereses. A tal fin, encuadran los temas que les conciernen con frame terms que resulten atractivos, para captar la atención de la opinión pública. A continuación, observan la respuesta que tiene esta estrategia y si resuena positivamente, intensifican sus esfuerzos en esa línea. Por el contrario, si la estrategia resuena negativamente, la parte interesada modifica la estrategia o la elimina del debate.

Aunque los frame terms no son los encuadres en sí mismos, representan los dispositivos de encuadre con los que éstos 
se manifiestan (Gamson; Modigliani, 1989; Van-Gorp; Vercruysse, 2012). De esta manera, las palabras y expresiones clave relacionadas con un tema son

"indicadores manifiestos a partir de los cuales se puede reconstruir la estructura latente y las dimensiones subyacentes del tratamiento informativo" (Igartua; Muñiz; Cheng, 2005, p. 158).

El análisis de las coocurrencias de los frame terms mediante técnicas de análisis de conglomerados proporciona un método fiable, riguroso y preciso para investigar los procesos de encuadre (Miller; Riechert, 2001, p. 119). Esta técnica es aplicable en situaciones en las que existen partes interesadas en articular posiciones en competencia para consolidar el apoyo de sus simpatizantes o para ganar nuevos adeptos (Miller, 1997, p. 368). Por tanto, es oportuna su implementación en estudios que analicen la comunicación política para medir su capacidad de influir en la cobertura informativa (Miller; Andsager; Riechert, 1998).

De acuerdo con la anterior conceptualización teórica y la fundamentación empírica, las características de nuestro caso de estudio y la naturaleza exploratoria del trabajo, proponemos las siguientes preguntas de investigación:

$\mathrm{PI}_{1}$ : ¿La cobertura de los periódicos reproduce los encuadres utilizados por los candidatos durante el debate?

$\mathrm{PI}_{2}$ : ¿Existen diferencias en la cobertura de los encuadres según el periódico?

\section{Metodología}

Para responder a la $\mathrm{PI}_{1}$ y averiguar qué frame terms utilizan los candidatos y cuál es su repercusión en la cobertura mediática, implementamos una metodología de análisis de contenido cuantitativo en dos fases, basada en la técnica del frame mapping (Miller, 1997). Mediante el programa de análisis de texto WordStat, aplicamos procedimientos estadísticos para, en primer lugar, extraer los encuadres utilizados por los candidatos en el debate electoral y, en segundo lugar, poder rastrear su presencia en la cobertura mediática.

En la primera fase, para elaborar la lista de frame terms que utilizan los candidatos, extraemos la lista de palabras utilizadas en el debate por cada candidato, descartando las palabras gramaticales y los términos que no están relacionados sustantivamente con el contexto (Lawlor, 2015; Miller,1997; Miller; Ansager; Riechert, 1998). En el caso de que encontremos palabras que posean más de un significado, se pueden desambiguar inspeccionando el contexto de la frase donde aparecen y añadiéndoles etiquetas para que el programa compute por separado cada una de sus acepciones. En el estudio etiquetamos los términos que pueden tener distintas acepciones (ciudadanos/Ciudadanos, podemos/Podemos, etc.), para que el programa sea capaz de computarlos correctamente.

A continuación, realizamos un análisis de correspondencias múltiple, siguiendo el procedimiento metodológico para la extracción de encuadres utilizado en investigaciones previas (Lawlor, 2015; Luther; Miller, 2005; Miller, 1997; Miller; Andsager; Riechert, 1998; Miller; Riechert, 2001). Este análisis permite construir una matriz tridimensional con los va- lores de los eigenvectores de las palabras utilizadas durante el debate, para establecer los frame terms que aparecen en el repertorio de un solo candidato y lo diferencian del discurso del resto.

Una vez delimitado el conjunto de frame terms que caracterizan el discurso de cada candidato, los agrupamos según la clasificación de marcos partidistas que proponen Valera, Carratalá y Palau (2017):

- En la senda del crecimiento y Mantener el rumbo (PP);

- Crisis multiorgánica y Seguir modernizando España (PSOE);

- La traición de las élites y Un gobierno de la gente (Podemos); y

- El bipartidismo agotado y Unidos en la regeneración (Ciudadanos).

Los autores definen este catálogo de encuadres después de realizar un análisis del discurso utilizado por los cuatro partidos en las publicaciones de sus respectivas webs durante la campaña electoral de 2015.

Para comprobar la fiabilidad y la validez del procedimiento de clasificación de encuadres, realizamos un análisis de conglomerados jerárquico, que nos permite establecer mediante un dendrograma los encuadres que coocurren en el discurso de cada candidato. Para definir el grado de proximidad de las coocurrencias se calcula el coeficiente de Jaccard, que compara la frecuencia en que dos palabras coocurren dentro de un mismo párrafo frente a la frecuencia en que aparecen separadas (Tan; Steinbach; Kumar, 2006). De esta manera, los valores próximos a la unidad indican que la coocurrencia entre las palabras es perfecta, mientras que el cero significa que nunca ocurren juntas.

En la segunda fase, examinamos las noticias publicadas en los periódicos para observar el nivel de penetración de los encuadres. Mediante la función de búsqueda de coincidencias con el diccionario del programa WordStat, medimos la presencia en la cobertura mediática de los frame terms utilizados por los cuatro candidatos, agrupados en los ocho encuadres partidistas. Para responder a la $\mathrm{PI}_{1}$, realizamos la prueba $t$ de Student para muestras independientes, que nos permite comprobar el impacto del debate en la prensa y establecer si existen diferencias significativas respecto de la cobertura anterior. De este modo, averiguamos si los encuadres definidos por los candidatos resuenan con mayor intensidad en la cobertura mediática tras el debate.

Por último, para responder a la $\mathrm{PI}_{2}$ realizamos una prueba Chi-cuadrado que permite establecer si existen diferencias significativas en la cantidad de noticias que protagoniza cada político. Adicionalmente, implementamos un análisis de correspondencias múltiple para comprobar si existen diferencias en el nivel de resonancia de los frame terms según el periódico que publica la noticia.

\subsection{Muestra}

Dado que buscamos establecer si se produce una transferencia a la cobertura mediática de los encuadres utilizados durante el debate electoral, disponemos de sendos corpus:

- el debate televisado, y 
- las noticias publicadas en la prensa.

El primer corpus está formado por la trascripción del debate electoral titulado 7D: El debate decisivo, emitido en directo el 7 de diciembre de 2015 por el grupo Atresmedia. En el debate participaron representantes de los cuatro partidos políticos con mayores expectativas para las elecciones generales españolas de 2015: Pedro Sánchez (PSOE), Pablo Iglesias (Podemos) y Albert Rivera (Ciudadanos) y Soraya Sáenz de Santamaría $(P P)$. Los tres primeros fueron al debate en calidad de candidatos de sus respectivos partidos, mientras que la última acudió en representación del $P P$, ya que su candidato, Mariano Rajoy, declinó participar en el debate. No obstante, nos referiremos a los cuatro políticos que participaron en el debate como candidatos para facilitar la redacción del trabajo.

El segundo corpus lo componen las noticias publicadas durante la semana anterior $(01 / 12 / 2015-07 / 12 / 2015)$ y posterior al debate (08/12/2015-14/12/2015) por las cuatro principales cabeceras españolas de distribución nacional, según el resumen general de resultados del $1^{r}$ Año Móvil 2016 del Estudio General de Medios: El país, El mundo, La vanguardia y $A B C$. De esta forma, podemos comparar los resultados del estudio durante las dos fases. El corpus de los medios lo descargamos de la base de datos Factiva, que ofrece una versión digitalizada del contenido de las noticias que contienen en el titular alguno de los nombres de los cuatro participantes en el debate, junto con el nombre del candidato del PP: Pedro Sánchez, Pablo Iglesias, Albert Rivera, Soraya Sáenz de Santamaría y Mariano Rajoy.

\subsection{Variables}

La matriz de datos final se elaboró incorporando todas las noticias en el programa QDA Miner, donde se definieron las siguientes variables: noticia, candidato, medio y fase.

- La variable noticia contiene el titular y el cuerpo de la información.

- La variable candidato indica el político que aparece en el titular: Mariano Rajoy $(P P)$, Soraya Sáenz de Santamaría $(P P)$, Pedro Sánchez (PSOE), Pablo Iglesias (Podemos) y Albert Rivera (Ciudadanos).

- La variable medio indica el periódico del que procede la noticia: El país, El mundo, La vanguardia y $A B C$.

- La variable fase indica si la noticia se ha publicado la semana anterior o posterior al debate.

\section{Resultados}

Con el fin de establecer el impacto del debate electoral del 7 de diciembre de 2015 en la cobertura mediática de los candidatos, se ha analizado la transcripción del programa para determinar los frame terms utilizados y buscar su presencia en las 506 noticias publicadas en los periódicos El país, $E l$ mundo, La vanguardia y $A B C$, que tienen en el titular el nombre de alguno de los participantes en el debate o del candidato del PP, Mariano Rajoy.

La tabla 1 muestra la distribución de frecuencias y porcentajes de los titulares protagonizados por los políticos según la fase cuando se publica la noticia. Mariano Rajoy es el político que acapara el porcentaje más elevado de titulares $(31,5 \%)$, seguido en orden decreciente por Pedro Sánchez $(23,5 \%)$, Albert Rivera $(20,4 \%)$, Pablo Iglesias (20\%) y Soraya Sáenz de Santamaría (3,4\%). Estos resultados señalan que existe una correlación entre la intensidad de la cobertura de cada candidato y la posición en el ranking electoral que pronostica el Barómetro preelectoral elecciones generales 2015, del Centro de Investigaciones Sociológicas (CIS, 2015) a sus respectivos partidos.

Como se observa en la tabla 1 , el número de noticias protagonizadas por los cinco políticos se duplica tras la celebración del debate. De este modo, se constata la relevancia informativa de este evento y su influencia en la composición de la agenda mediática. Este aumento se produce en la cobertura de todos los políticos excepto en el caso de Soraya Sáenz de Santamaría, que disminuye su visibilidad pese a su participación en el debate.

Para extraer los frame terms utilizados durante el debate, realizamos un análisis de correspondencias múltiple de las palabras sustantivas que encontramos en la transcripción de la intervención de cada candidato y los agrupamos en los ocho encuadres que han utilizado durante la campaña electoral de 2015 (Valera; Carratalá; Palau, 2017). El gráfico 1 nos ofrece una representación tridimensional del análisis de correspondencias, donde podemos comprobar que los encuadres aparecen concentrados en un punto muy próximo al nombre de cada político, lo que implica que el nivel de coocurrencia se circunscribe exclusivamente a sus respectivos discursos.

El gráfico 2 muestra el dendrograma con el índice de similaridad de los encuadres según el nivel de coocurrencia en los candidatos. El valor del coeficiente de Jaccard se aproxima a la unidad entre los encuadres del mismo partido, mien- 


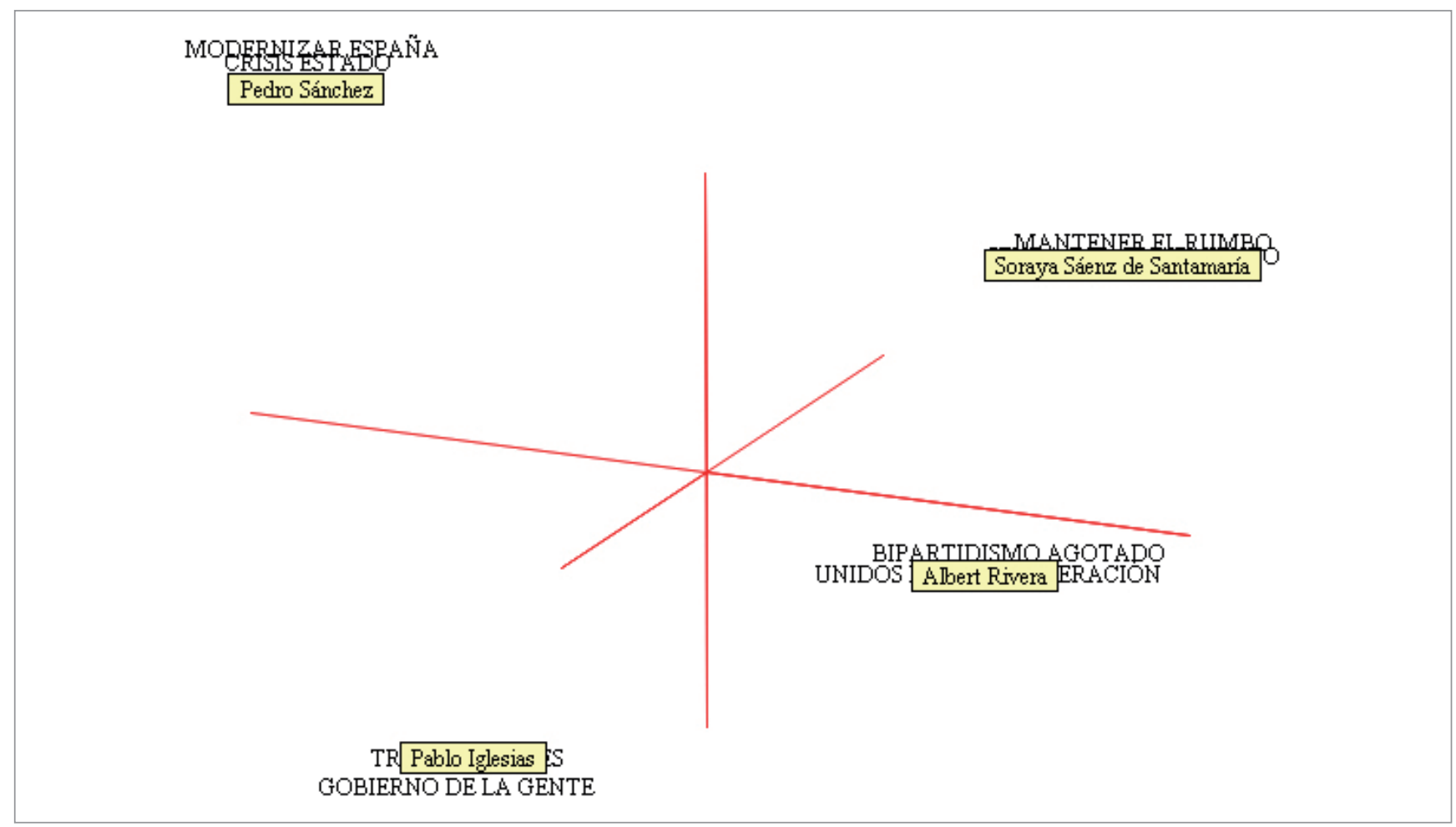

Gráfico 1. Análisis de correspondencias de los encuadres de los candidatos (elaborado con WordStat)

tras que entre los encuadres de distintos partidos el índice de similaridad es casi inapreciable. De este modo, podemos validar la clasificación de los frame terms en encuadres. En el caso de los encuadres de Podemos y Ciudadanos, el índice de similaridad es distinto a cero, aunque con un valor muy bajo $(J=0,036, p>0,000.001)$, debido a los frame terms compartidos en ambos partidos sobre regeneración democrática.

Para responder a la $\mathrm{PI}_{1}$, realizamos la prueba $t$ de Student para muestras independientes, que permite comprobar si existen diferencias estadísticamente significativas en la varianza de las medias de los frame terms de los candidatos que aparecen en la cobertura anterior y posterior del debate. La tabla 2 muestra la media y desviación estándar de los encuadres según la fase en que se publica la noticia. De los encuadres utilizados por los cuatro partidos, tan solo los utilizados por Pablo Iglesias experimentan un incremento estadísticamente significativo tras celebrarse el debate $[t(502,45)=-2,7, p=0,024]$, pasando de una media de 0,14 frame terms antes del debate a 0,28 después.

Si analizamos los ocho encuadres por separado, sólo encontramos un incremento estadísticamente significativo en el encuadre de Podemos, Traición de las élites $[t(489,37)=$ $-2,18, p=0,029]$. En este encuadre, la media de frame terms pasa de 0,07 a 0,18 después del debate. En los encuadres utilizados por el resto de candidatos durante el debate no encontramos un aumento significativo de su presencia en la cobertura mediática posterior.

Para responder a la $\mathrm{PI}_{2}$ y comprobar si existen diferencias en la cobertura mediática, realizamos análisis de correspondencias múltiple de los ocho encuadres según su coocurren-

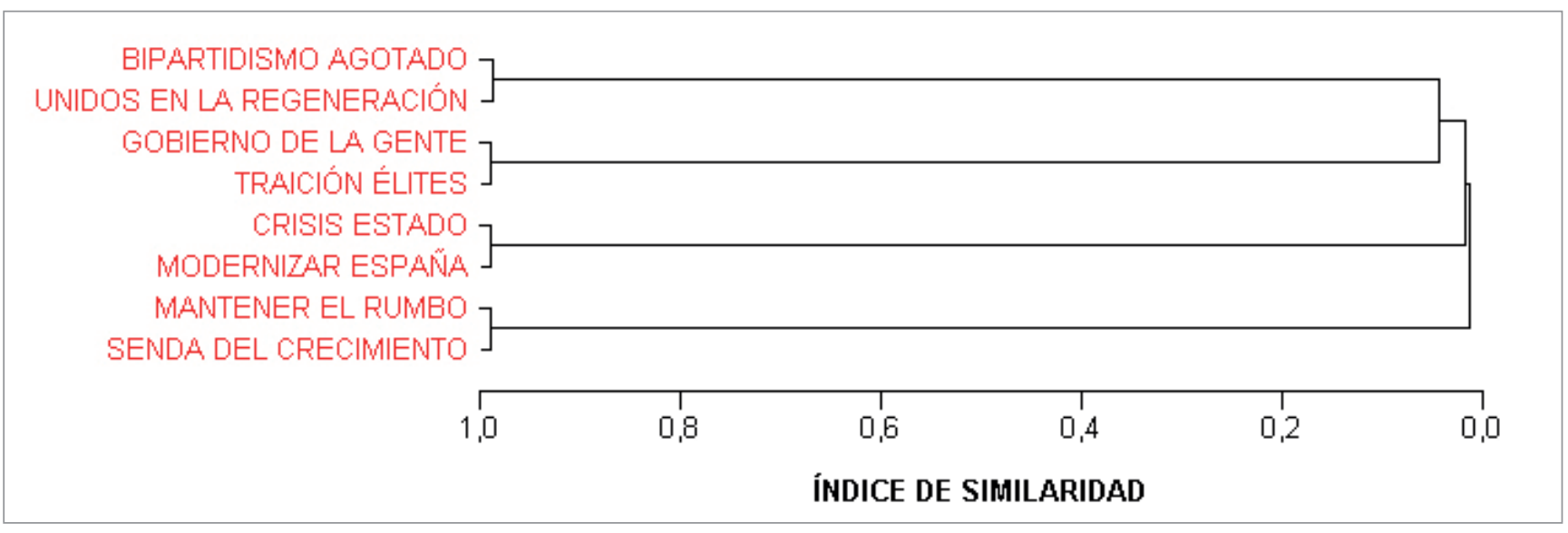

Gráfico 2. Dendrograma de los encuadres de los candidatos (elaborado con WordStat) 
Tabla 2. Media y desviación estándar según encuadre y fase

\begin{tabular}{|l|c|c|c|c|}
\cline { 2 - 5 } \multicolumn{1}{c|}{} & \multicolumn{4}{c|}{ Fase } \\
\cline { 2 - 5 } \multicolumn{1}{c|}{} & \multicolumn{2}{c|}{ Antes del debate } & \multicolumn{1}{c|}{$\begin{array}{c}\text { Después del } \\
\text { debate }\end{array}$} \\
\cline { 2 - 5 } & M & SD & M & SD \\
\hline Bipartidismo agotado & 0,01 & 0,078 & 0,01 & 0,108 \\
\hline Crisis de Estado & 0,01 & 0,078 & 0,00 & 0,000 \\
\hline Gobierno de la gente & 0,07 & 0,273 & 0,10 & 0,420 \\
\hline Mantener el rumbo & 0,02 & 0,134 & 0,01 & 0,121 \\
\hline Modernizar España & 0,06 & 0,285 & 0,02 & 0,142 \\
\hline Senda del crecimiento & 0,02 & 0,134 & 0,01 & 0,108 \\
\hline Traición élites & 0,07 & 0,322 & 0,18 & 0,807 \\
\hline Unidos en la regeneración & 0,22 & 0,518 & 0,16 & 0,592 \\
\hline
\end{tabular}

cia en los cuatro periódicos. La tabla 3 muestra la distribución de frecuencias y residuos tipificados corregidos de los titulares protagonizados por los políticos según el medio donde se publica la noticia. La prueba de Chi-cuadrado señala que existen diferencias estadísticamente significativas en la frecuencia de aparición de los políticos según el medio $\left[\chi^{2}(12,0 N=506)=26,72, p=0,008\right]$. Si atendemos al número de veces que encontramos a cada político, destaca que Mariano Rajoy acapara el porcentaje más elevado de titulares en los cuatro medios, como cabría esperar debido a que es el presidente saliente y el candidato con mejores resultados en las encuestas.

El análisis de los resultados de los residuos tipificados corregidos revela diferencias estadísticamente significativas en el porcentaje de noticias dedicadas a cada candidato. En el caso de Pedro Sánchez, el líder socialista aparece en los titulares de $E I$ mundo con una frecuencia inferior a la esperada $(Z=-2,2)$, mientras que en $A B C$ protagoniza un número de noticias superior al esperado $(Z=$ $2,4)$. El fenómeno inverso sucede con Albert Rivera, que recibe una atención menor de la esperada en $A B C(Z=-2,1)$ y mayor en $E l$ mundo $(Z=2,0)$. Por último, la representante del $P P$ que acudió al debate, Soraya Sáenz, apenas aparece en la cobertura mediática, sobre todo en el caso de $A B C(Z=-2,1)$. Destaca que La vanguardia y El país son los medios donde encontramos una distribución más equilibrada de candidatos en su cobertura, mientras que en El mundo y, especialmente, en $A B C$ sucede lo contrario.

En cuanto al contenido de las noticias, el gráfico 3 muestra una representación tridimensional del análisis de correspondencias, donde la disposición es 2,55 espacial de cada encuadre en relación con el nombre del periódico nos ofrece una idea de las estrategias seguidas en la cobertura de la campaña.

$L a$ vanguardia y $A B C$ son los periódicos que mayores diferencias muestran en la cobertura de los temas utilizados por los políticos: el primero privilegia en sus noticias el encuadre de Podemos, Gobierno de la gente; mientras que el segundo abunda en los encuadres del Partido Popular (Senda del crecimiento y Mantener el rumbo) y del Partido Socialista (Crisis de Estado). La situación espacial de estos dos medios en el análisis de correspondencias revela una oposición sustancial en la selección de los encuadres de los candidatos.

En cambio, El país y El mundo realizan una cobertura de los encuadres más parecida, que se refleja en el análisis de correspondencias en una posición más próxima y centrada. No obstante, encontramos pequeñas diferencias en la selección de los encuadres: El mundo se centra en temas relacionados con la corrupción, destacando los encuadres regeneracionistas de Podemos (Traición de las élites) y Ciudadanos (Unidos en la regeneración); por su parte, El país, aunque incorpora algunos elementos del discurso de Ciudadanos (Unidos en la regeneración), se caracteriza por estar más próximo al encuadre del PSOE (Modernizar España).

\section{Conclusiones}

Los debates electorales televisados son importantes para el sistema democrático porque ofrecen la oportunidad a los votantes de escuchar directamente a los candidatos (Luengo, 2011) y valorar su calificación para el cargo en disputa (Chihu,

Tabla 3. Frecuencia y porcentajes de noticias según protagonista del titular y medio*

\begin{tabular}{|c|c|c|c|c|c|c|}
\hline & & & \multicolumn{4}{|c|}{ Medio } \\
\hline & & & $A B C$ & Elmundo & El país & $\begin{array}{l}\text { La van- } \\
\text { guardia }\end{array}$ \\
\hline \multirow{15}{*}{ Protagonista } & \multirow{3}{*}{ Albert Rivera } & Recuento & 28 & 34 & 29 & 12 \\
\hline & & $\%$ & $15,3 \%$ & $26,6 \%$ & $24,4 \%$ & $15,8 \%$ \\
\hline & & Residuos & $-2,1$ & 2,0 & 1,2 & $-1,1$ \\
\hline & \multirow{3}{*}{ Mariano Rajoy } & Recuento & 68 & 35 & 34 & 29 \\
\hline & & $\%$ & $37,2 \%$ & $27,3 \%$ & $28,6 \%$ & $38,2 \%$ \\
\hline & & Residuos & 1,6 & $-1,5$ & $-1,1$ & 1,1 \\
\hline & \multirow{3}{*}{ Pablo Iglesias } & Recuento & 31 & 32 & 22 & 16 \\
\hline & & $\%$ & $16,9 \%$ & $25,0 \%$ & $18,5 \%$ & $21,1 \%$ \\
\hline & & Residuos & $-1,3$ & 1,7 & $-0,5$ & 0,3 \\
\hline & \multirow{3}{*}{ Pedro Sánchez } & Recuento & 54 & 21 & 26 & 18 \\
\hline & & $\%$ & $29,5 \%$ & $16,4 \%$ & $21,8 \%$ & $23,7 \%$ \\
\hline & & Residuos & 2,4 & $-2,2$ & $-0,5$ & 0,0 \\
\hline & \multirow{3}{*}{ Soraya Sáenz } & Recuento & 2 & 6 & 8 & 1 \\
\hline & & & $1,1 \%$ & $4,7 \%$ & $6,7 \%$ & $1,3 \%$ \\
\hline & & Residuos & $-2,1$ & 1,0 & 2,3 & $-1,1$ \\
\hline \multirow{2}{*}{\multicolumn{2}{|c|}{ Total }} & Recuento & 183 & 128 & 119 & 76 \\
\hline & & $\%$ & $100 \%$ & $100 \%$ & $100 \%$ & $100 \%$ \\
\hline
\end{tabular}

* Tres casillas (15\%) tienen una frecuencia esperada inferior a 5. La frecuencia mínima esperada 


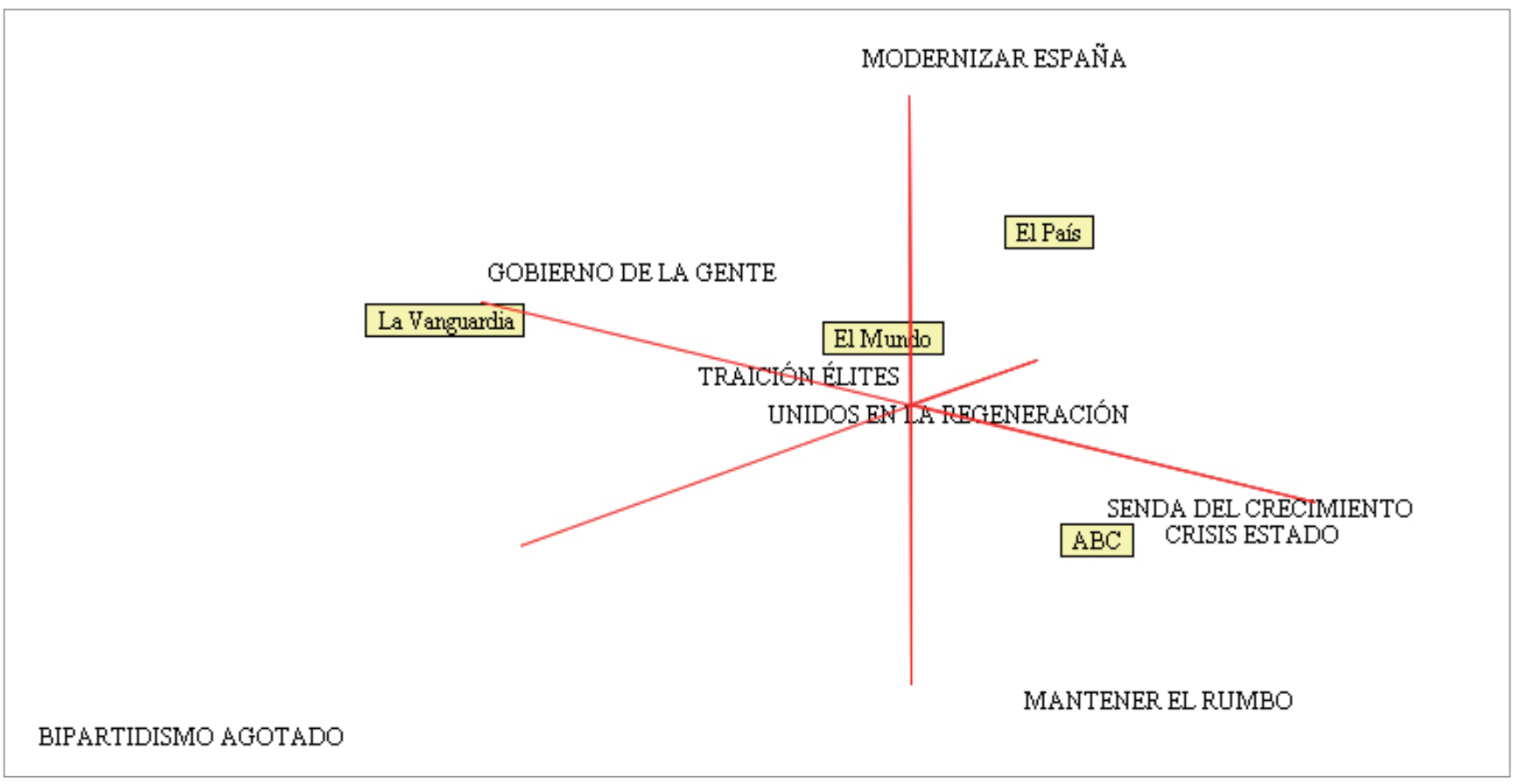

Gráfico 3. Análisis de correspondencias de los encuadres según el periódico (elaborado con WordStat)

2008). Estos adquieren mayor visibilidad gracias a su cobertura en redes sociales y otros medios de comunicación, como es el caso del "debate del debate" que se realiza en la prensa, donde se determina qué ha sido lo más relevante (Llorca-Abad; López-García, 2016). Sin embargo, los medios centran su cobertura en elementos que están asociados al debate pero no a su contenido (Benoit; Currie, 2001; García-Marín, 2015; Martín-Jiménez; Reguero-Sanz; Velasco-Molpeceres, 2015).
Los resultados del estudio muestran que los debates juegan un papel importante en la configuración de la agenda de la prensa escrita, con un incremento considerable de noticias sobre los candidatos en los días posteriores a su celebración. Sin embargo, el tratamiento informativo del debate se centra en establecer quién ha ganado y, salvo excepciones, deja al margen los temas y encuadres utilizados por los participantes.

GANAR

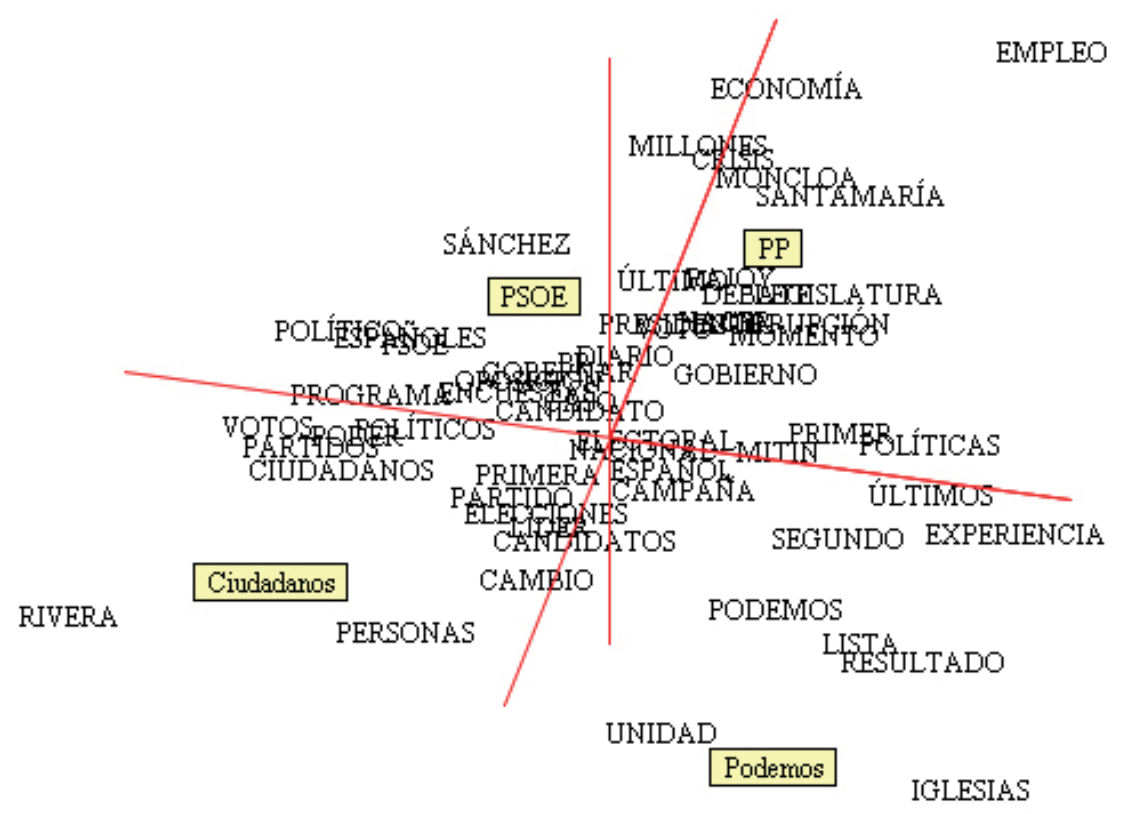

Gráfico 4. Análisis de correspondencias de las palabras más utilizadas por los medios según el partido del protagonista del titular (elaborado con WordStat) 
En consonancia con las conclusiones que arrojan otros estudios, los medios analizados abordan la cobertura de la campaña electoral con noticias que privilegian el encuadre de juego (Berganza, 2008; Cappella; Jamieson, 1997; De-Vreese, 2005; Lawrence, 2010; López-Rabadán, 2010; Muñiz, 2015; Strömbäck; Luengo, 2008). Como se puede observar en el gráfico 4, entre las 50 palabras más utilizadas por los medios para informar durante la campaña, se encuentra un gran repertorio de términos para describir la posición del candidato en la carrera electoral desde una perspectiva deportiva: ganar, resultado, sondeos, líder, primer, primero, segundo, último, últimos, etc.

Asimismo, la forma en que los periódicos reproducen los encuadres de los candidatos revela el posicionamiento estratégico de cada cabecera ante las diversas opciones políticas: $A B C$ confronta los encuadres de los grandes partidos, aunque centrado principalmente en los del Partido Popular; El país privilegia los mensajes del PSOE; El mundo hace especial hincapié en los encuadres sobre la regeneración democrática y la lucha contra la corrupción de los nuevos partidos; por último, La vanguardia incorpora de manera notable los frame terms de Podemos relacionados con el gobierno de la gente y el derecho a decidir de los catalanes. De esta forma, los medios no confrontan las propuestas de todos los candidatos, sino que hacen una reproducción selectiva de los encuadres coincidentes con su línea editorial.

Los hallazgos encontrados constatan el alejamiento de los medios de su función normativa de proveer información acerca de los acontecimientos y sus contextos (Christians; Glasser; McQuail; White, 2009). La cobertura mediática de los debates electorales está constreñida por la orientación de cada medio y ofrece un escenario en el que se presenta a los candidatos como si participaran en una carrera de caballos (Aalberg; Strömback; De-Vreese, 2011; lyengar; Norpoth; Hahn, 2004; Reinemann; Wilke, 2007; Rosenstiel; Kovach, 2001; Patterson, 1994). La omisión de los temas de fondo abordados por los políticos y la simplificación de la información al binomio ganador/perdedor aleja a los medios de este ideal normativo y priva a la audiencia de los elementos de juicio necesarios para una elección de voto fundamentada. En este sentido, futuros estudios deberían ampliar el período de análisis y cuantificar el encuadre de juego para comprobar si es consustancial al tratamiento de la información política o sólo aparece durante la cobertura electoral.

\section{Nota}

Este artículo forma parte de la producción académica del proyecto de I+D+i "Los flujos de comunicación en los procesos de movilización política: medios, blogs y líderes de opinión" (ref. CSO2013-43960-R).

\section{Referencias}

Aalberg, Toril; Strömbäck, Jesper; De-Vreese, Claes H. (2011). "The framing of politics as strategy and game: A review of concepts, operationalizations and key findings". Journalism, v. 13, n. 2, pp. 162-178.

https://doi.org/10.1177/1464884911427799

Arcila-Calderón, Carlos; Barbosa-Caro, Eduar; Cabezuelo-Lo- renzo, Francisco (2016). "Técnicas big data: análisis de textos a gran escala para la investigación científica y periodística". El profesional de la información, v. 25, n. 4, pp. 623-631. https://doi.org/10.3145/epi.2016.jul.12

Benoit, William L.; Currie, Heather (2001). "Inaccuracies in media coverage of the 1996 and 2000 presidential debates". Argumentation and advocacy, v. 38, n. 1, pp. 28-39. https://goo.gl/3pRzDS

Berganza, María-Rosa (2008). "Las elecciones al Parlamento Europeo como comicios de segundo orden. Estudio de la cobertura informativa en televisión (1999-2004)". Estudios sobre el mensaje periodístico, v. 14, pp. 15-31.

http://revistas.ucm.es/index.php/ESMP/article/view/ ESMP0808110015A

Cacciatore, Michael A.; Scheufele, Dietram A.; Iyengar, Shanto (2016). "The end of framing as we know it... and the future of media effects". Mass communication and society, v. 19, n. 1, pp. 7-23.

https://doi.org/10.1080/15205436.2015.1068811

Cappella, Joseph N.; Jamieson, Kathleen Hall (1997). Spiral of cynicism: The press and the public good. Oxford: University Press. ISBN: 9780195090642

Chihu, Aquiles (2008). "El framing de los debates presidenciales de 2006 en México". Cultura y representaciones sociales, v. 3, n. 5, pp. 125-155.

Chihu, Aquiles (2011). El framing de la prensa. México: UAM-I. ISBN: 9786074773767

Chong, Dennis; Druckman, James N. (2007). "Framing theory". Annual review of political science, v. 10, n. 2, pp. 103-26. https://doi.org/10.1146/annurev.polisci.10.072805.103054 http://www.annualreviews.org/doi/pdf/10.1146/annurev. polisci.10.072805.103054

Christians, Clifford G.; Glasser, Theodore; McQuail, Denis; White, Robert (2009). Normative theories of the media. Journalism in democratic societies. Illinoils: University of Illinois Press. ISBN: 9780252076183

CIS (Centro de Investigaciones Sociológicas). Barómetro preelectoral elecciones generales 2015 (2015). http://www.cis.es/cis/export/sites/default/-Archivos/ Marginales/3100_3119/3117/Es3117mar.pdf

D’Angelo, Paul (2002). "News framing as a multiparadigmatic research program: A response to Entman". Journal of communication, v. 52, n. 4, pp. 870-888. https://doi.org/10.1093/joc/52.4.870

De-Vreese, Claes H. (2005). "The spiral of cynicism reconsidered". European journal of communication, v. 20, n. 3, pp. 283-301.

https://doi.org/10.1177/0267323105055259

De-Vreese, Claes H. (2012). "New avenues for framing research". American behavioral scientist, v. 56, n. 3, pp. 365-375. https://doi.org/10.1177/0002764211426331

Entman, Robert (1991). "Symposium framing US coverage of international news: Contrasts in narratives of the KAL and Iran air incidents". Journal of communication, v. 41, n. 4, pp. 6-27. 
Entman, Robert (1993). "Framing: Toward clarification of a fractured paradigm". Journal of communication, v. 43, n. 4, pp. 51-58.

Entman, Robert (2010). “Media framing biases and political power: Explaining slant in news of Campaign 2008". Journalism, v. 11, n. 4, pp. 389-408.

https://doi.org/10.1177/1464884910367587

Fenoll, Vicente (2015). "Segundo nivel de agenda-setting y medios digitales: estudio de la transferencia de atributos en el sistema de comentarios". En: Actas del VII Congreso internacional de ciberperiodismo y web 2.0 2015, 9-10 de noviembre, Univ. del País Vasco, pp. 83-102. ISBN: 97884 90822364

http://www.ehu.eus/documents/3399833/0/Actas_Aktak ConferenceProceedings_VIICiberpebi.pdf

Fenoll, Vicente (2016). "Estudios de framing mediante análisis textual asistido por computadora". En: Actas del III Congreso de Incom-Chile 2016, 4 nov., Univ. Católica de la Sma. Concepción (Chile).

Gamson, William A.; Modigliani, Andre (1989). "Media discourse and public opinion on nuclear power: A constructionist approach". American journal of sociology, v. 95, n. 1, pp. 1-37.

http://www.jstor.org/stable/2780405

García-Marín, Javier (2015). "La cobertura mediática de los debates electorales en España”. Revista española de ciencia política, n. 38, pp. 135-161.

http://recyt.fecyt.es/index.php/recp/article/viewFile/37666/21183

Groshek, Jacob; Engelbert, Jiska (2013). “Double differentiation in a cross-national comparison of populist political movements and online media uses in the United States and the Netherlands". New media \& society, v. 15, n. 2, pp. 183-201. https://doi.org/10.1177/1461444812450685

Grundmann, Reiner; Krishnamurthy, Ramesh (2010). "The discourse of climate change: a corpus-based approach". Critical approaches to discourse analysis across disciplines, v. 4, n. 2, pp. 125-146.

http://acorn.aston.ac.uk/RK-publications/2010-CADAAD_ Grundmann-and-Krishnamurthy.pdf

Harwood, Tracy; Garry, Tony (2003). "An overview of content analysis". The marketing review, v. 3, pp. 479-498. https://doi.org/10.1362/146934703771910080

Igartua, Juan-José; Muñiz, Carlos; Cheng, Lifen (2005). “La inmigración en la prensa española. Aportaciones empíricas y metodológicas desde la teoría del encuadre noticioso". Migraciones. Publicación del Instituto Universitario de Estudios sobre Migraciones, v. 17, pp. 143-181.

http://revistas.upcomillas.es/index.php/revistamigraciones/ article/view/4220

Iyengar, Shanto; Norpoth, Helmut; Hahn, Kyu S. (2004). "Consumer demand for election news: The horserace sells". The journal of politics, v. 66, n. 1, pp. 157-175.

https://doi.org/10.1046/j.1468-2508.2004.00146.x

Just, Sine-Nørholm (2009). "No place like home? The role of the media in the framing of Europe". Journal of language and politics, v. 8, n. 2, pp. 244-268. https://doi.org/10.1075/jlp.8.2.04jus

https://benjamins.com/\#catalog/journals/jlp.8.2.04jus/details

Lawlor, Andrea (2015). "Framing immigration in the Canadian and British news media". Canadian journal of political science, v. 48, n. 2, pp. 329-355.

https://doi.org/10.1017/S0008423915000499

http://journals.cambridge.org/article_S0008423915000499

Lawrence, Regina G. (2010). "Researching political news framing". En: D’Angelo, Paul; Kuypers, Jim A. (eds.), Doing news framing analysis: Empirical and theoretical perspectives (pp. 265-285). London: Routledge. ISBN: 978 0415992350

https://doi.org/10.4324/9780203864463

Llorca-Abad, Germán; López-García, Guillermo (2016). “Los debates televisados de la campaña de las Elecciones Generales de 2015 en España". Comunicación presentada en el congreso La nueva comunicación y los procesos de movilización política: partidos, medios y ciudadanos, 16-18 de noviembre, Valencia (UIMP y Universidad de Valencia).

López-Rabadán, Pablo (2010). "Nuevas vías para el estudio del framing periodístico. La noción de estrategia de encuadre". Estudios sobre el mensaje periodístico, v. 16, pp. 235-258.

http://revistas.ucm.es/index.php/ESMP/article/view/ ESMP1010110235A

Luther, Catherine A.; Miller, M. Mark (2005). “Framing of the 2003 US-Iraq war demonstrations: An analysis of news and partisan texts". Journalism \& mass communication quarterly, v. 82, n. 1, pp. 78-96.

https://doi.org/10.1177/107769900508200106

Martín-Jiménez, Virginia; Reguero-Sanz, Itziar; VelascoMolpeceres, Ana (2015). "El debate del debate: 'Salvados' y el politainment como generador de contenidos en la prensa tradicional". En: Mateos, Concha; Herrero, Javier (coords.), La pantalla insomne. Cuadernos artesanos de comunicación 90. (pp. 1449-1467). La Laguna: Ed. Latina de Comunicación Social. ISBN: 9788415698982.

https://doi.org/10.4185/cac90

http://www.revistalatinacs.org/15SLCS/2015_libro/068 Martin.pdf

Matthes, Jörg (2012). "Framing politics: An integrative approach". American behavioral scientist, v. 56, n. 3, pp. 247-259. https://doi.org/10.1177/0002764211426324

Matthes, Jörg (2014). Framing. Baden-Baden: Nomos. ISBN: 9783832959661

Matthes, Jörg; Kohring, Matthias (2008). "The content analysis of media frames: Toward improving reliability and validity". Journal of communication, v. 58, n. 2, pp. 258-279. https://doi.org/10.1111/j.1460-2466.2008.00384.x

Miller, M. Mark (1997). "Frame mapping and analysis of news coverage of contentious issues". Social science computer review, v. 15, n. 4, pp. 367-378.

Miller, M. Mark; Andsager, Julie L.; Riechert, Bonnie-Parnell (1998). "Framing the candidates in presidential primaries: Issues and images in press releases and news coverage". Journalism \& mass communication quarterly, v. $75, \mathrm{n}$. 2, pp. 312-324. 
Miller, M. Mark; Riechert, Bonnie-Parnell (2001). "The spiral of opportunity and frame resonance: Mapping the issue cycle in news and public discourse". En: Reese, Stephen D.; Grant, August E.; Gandy, Oscar H. (eds.) Framing public life: Perspectives on media and our understanding of the social world, pp. 107-121. Mahwah, NJ: Erlbaum. ISBN: 9781 410605689.

Muñiz, Carlos (2015). “La política como debate temático o estratégico. Framing de la campaña electoral mexicana de 2012 en la prensa digital". Comunicación y sociedad, v. 23, pp. 67-95.

https://goo.gl/vjMtif

Neuendorf, Kimberly (2002). The content analysis guidebook. Sage. ISBN: 9781412979474

Pan, Zhongdang; Kosicki, Gerald M. (1993). "Framing analysis: An approach to news discourse". Political communication, v. 10 , n. 1 , pp. 55-75.

Patterson, Thomas E. (1994). Out of order: An incisive and boldly original critique of the news media's domination of America's political process. Nueva York: Vintage. ISBN: 978 0679755104

Patterson, Thomas E. (1996). "Bad news, bad governance". The annals of the American Academy of Political and Social Science, v. 546, n. 1, pp. 97-108.

https://doi.org/10.1177/0002716296546001009

Patterson, Thomas E. (1998). “Time and news: The media's limitation as an instrument of democracy". International political science review, v. 19, n. 1, pp. 55-67. https://doi.org/10.1177/019251298019001004

Reinemann, Carsten; Wilke, Jürgen (2007). "It's the debates, stupid! How the introduction of televised debates changed the portrayal of chancellor candidates in the German press, 1949-2005". The international journal of press/politics, v. 12, n. 4, pp. 92-111.

https://doi.org/10.1177/1081180X07307185

Rosenstiel, Tom; Kovach, Bill (2001). The elements of journalism. New York: Crown. ISBN: 9780804136785

Scheufele, Dietram A.; Iyengar, Shanto (2014). "The state of framing research: A call for new directions". En: Kenski, Kate; Jamieson, Kathleen Hall (eds.). The Oxford handbook of political communication, pp. 1-26. New York: Oxford University Press.

Soroka, Stuart; Lawlor, Andrea; Farnsworth, Stephen; Young, Lori (2012). "Mass media and policymaking". En: Araral, Eduardo; Fritzen, Scott; Howlett, Michael; Ramesh, M.; Wu, Xun (eds.), Routledge handbook of public policy. Abingdon, UK: Routledge. ISBN: 9780415782456

Strömbäck, Jesper; Luengo, Oscar G. (2008). “Polarized pluralist and democratic corporatist models. A comparison of election news coverage in Spain and Sweden". International communication gazette, v. 70, n. 6, pp. 547-562. https://doi.org/10.1177/1748048508096398

Tan, Pang-Ning; Steinbach, Michael; Kumar, Vipin (2006). Introduction to data mining. Boston, MA: Pearson Addison Wesley. ISBN: 9781292026152

Tankard, James (2001). "The empirical approach to the study of media framing". En: Reese, Stephen D.; Gandy, Oscar H.; Grant, August E., Framing public life: Perspectives of media and our understanding of the social world (pp. 95106). Mahwah, NJ: Erlbaum.

Tolley, Erin (2011). "Racial mediation and the framing of candidates' viability in Canadian politics". En: $6^{\text {th }}$ General conf of the European Consortium for Political Research. http://ecpr.eu/filestore/paperproposal/c3bebd9a-932e48a3-bd2c-4ab42de4c3da.pdf

Touri, Maria; Koteyko, Nelya (2015). "Using corpus linguistic software in the extraction of news frames: Towards a dynamic process of frame analysis in journalistic texts". International journal of social research methodology, v. 18, n. 6, pp. 601-616.

http://www.tandfonline.com/doi/abs/10.1080/13645579.2 014.929878

https://doi.org/10.1080/13645579.2014.929878

Valera, Lidia; Carratalá, Adolfo; Palau, Dolors (2017). “La batalla de los partidos por la definición de la realidad social: los marcos partidistas durante las Elecciones Generales 2015". En: López-García, Guillermo; Valera-Ordaz, Lidia (eds.), Pantallas electorales. El discurso de partidos, medios y ciudadanos en la campaña de 2015 (pp. 195-207). Valencia: Editorial UOC. ISBN: 9788491167051

Van-Gorp, Baldwin; Vercruysse, Tom (2012). "Frames and counter-frames giving meaning to dementia: A framing analysis of media content". Social science \& medicine, n. 74, pp. 1274-1281.

https://doi.org/10.1016/j.socscimed.2011.12.045

Vicente-Mariño, Miguel; López-Rabadán, Pablo (2009). "Resultados actuales de la investigación sobre framing: sólido avance internacional y arranque de la especialidad en España". ZER - Revista de estudios de comunicación, v. 14, n. 26, pp. 13-34.

http://www.ehu.eus/ojs/index.php/Zer/article/view/2750

White, Marilyn-Domas; Marsh, Emily E. (2006). "Content analysis: A flexible methodology". Library trends, v. 55, n. 1, pp. 22-45.

https://www.ideals.illinois.edu/bitstream/handle/2142/3670/ whitemarch551.pdf?sequense $=2$

https://doi.org/10.1353/lib.2006.0053

Young, Lori; Soroka, Stuart (2012). "Affective news: The automated coding of sentiment in political texts". Political communication, v. 29, n. 2, pp. 205-231.

https://doi.org/10.1080/10584609.2012.671234 